\title{
Simplified Sum-Over-States Approach for Predicting Resonance Raman Spectra. Application to Nucleic Acid Bases
}

\section{Citation}

Rappoport, Dmitrij, Sangwoo Shim, and Alan Aspuru-Guzik. 2011. Simplified sum-over-statesapproach for predicting resonance Raman spectra. Application to Nucleic Acid Bases. Journal of Physical Chemistry Letters 2(11): 1254-1260.

\section{Published Version}

doi:10.1021/jz200413g

\section{Permanent link}

http://nrs.harvard.edu/urn-3:HUL.InstRepos:8365128

\section{Terms of Use}

This article was downloaded from Harvard University's DASH repository, and is made available under the terms and conditions applicable to Open Access Policy Articles, as set forth at http:// nrs.harvard.edu/urn-3:HUL.InstRepos:dash.current.terms-of-use\#OAP

\section{Share Your Story}

The Harvard community has made this article openly available.

Please share how this access benefits you. Submit a story.

\section{Accessibility}




\title{
A Simplified Sum-Over-States Approach for
}

\section{Predicting Resonance Raman Spectra. Application to Nucleic Acid Bases}

\author{
Dmitrij Rappoport,* Sangwoo Shim, and Alán Aspuru-Guzik* \\ Department of Chemistry and Chemical Biology, Harvard University, 12 Oxford Street, \\ Cambridge MA, 02138, USA
}

E-mail: rappoport@chemistry.harvard.edu; aspuru@chemistry.harvard.edu

*To whom correspondence should be addressed 


\begin{abstract}
Resonance Raman spectra provide a valuable probe into molecular excited-state structures and properties. Moreover, resonance enhancement is of importance for the chemical contribution to surface-enhanced Raman scattering. In this work, we introduce a simplified sum-overstates scheme for computing Raman spectra and Raman excitation profiles. The proposed sumover-states approach uses derivatives of electronic excitation energies and transition dipole moments, which can be efficiently computed from time-dependent density functional theory. We analyze and interpret the resonance Raman spectra and Raman excitation profiles of nucleic acid bases using the present approach. Contributions of individual excited states under strictly resonant and non-resonant conditions are investigated and smooth interpolation between both limiting cases is obtained.
\end{abstract}

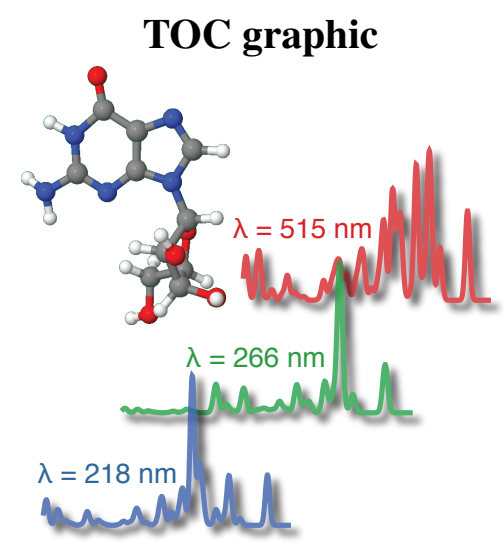

Keywords: Resonance Raman spectra, density functional theory, vibronic coupling, electronic excitations, nucleic acid bases.

Resonance enhancement of Raman scattering, which occurs whenever the frequency of the incident radiation approaches molecular excitation frequencies, was reported some 20 years after the initial experimental observation of the Raman effect. ${ }^{1,2}$ The large degree of enhancement spanning several orders of magnitude is useful for detection of the inherently inefficient spontaneous Raman scattering. Moreover, the shapes of Raman spectra change considerably at resonance with molecular excitations and provide information on structures and properties of electronic excited states. Resonance Raman spectroscopy is a sensitive spectroscopic technique for strongly 
absorbing chemical constituents such as nucleic acid bases, aromatic aminoacids, and heme chromophores $^{3-5}$

Another important manifestation of resonance enhancement emerges in surface-enhanced Raman scattering (SERS). ${ }^{6-8}$ The surface enhancement of Raman scattering is observed in molecules adsorbed on rough or nanostructured noble-metal surfaces and comprises an electromagnetic and a chemical contribution. ${ }^{8,9}$ The chemical contribution to the SERS intensities, while generally smaller in magnitude than the electromagnetic enhancement, is sensitive to the electronic structure of the adsorbate. The chemical effect leads to characteristic changes in the relative intensities of Raman bands and alters the overall shape of the Raman spectra compared to neat substance. Chemical effects are satisfactorily described by cluster models and can be attributed to resonance enhancement due to interface states. ${ }^{10,11}$ The combination of surface enhancement with intramolecular excitations gives rise to surface-enhanced resonance Raman scattering (SERRS) which provides an extraordinary sensitivity, even to the level of single-molecule detection. ${ }^{12-14}$

While theoretical descriptions of resonance Raman scattering has been developed early on by Shorygin and co-workers ${ }^{2,15}$ and by Albrecht, ${ }^{16,17}$ calculations of resonance Raman scattering from medium-size and large molecules are not often routinely performed. Raman scattering is a second-order process and its cross sections are given by the Kramers-Heisenberg-Dirac (KHD) dispersion relation. ${ }^{18,19}$ The classical expression for Raman cross sections involving derivatives of electronic polarizabilities with respect to vibrational normal modes can be obtained via closure of the sum over intermediate vibronic states in the KHD expression. ${ }^{20,21}$ The description of Shorygin and co-workers ${ }^{15,22}$ represents the polarizability derivatives as a sum over electronic states and introduces parameters for the resulting derivatives of excitation energies and oscillator strengths of the lowest excited state with respect to vibrational normal modes.

On the other hand, Albrecht's approach is rooted in the vibronic coupling theory ${ }^{23,24}$ and introduces the Herzberg-Teller expansion into the sum over vibronic states of the KHD dispersion relation. Each vibronic state contributes four different terms denoted A, B, C, and D by Albrecht. The A term is due to vibrational wavefunction overlap of the initial and the intermediate state and 
of the intermediate and the final state. The $\mathrm{B}$ and $\mathrm{C}$ terms arise from the dependence of transition dipole moments on vibrational coordinates and are analogous to the intensity borrowing terms of vibronic coupling theory. ${ }^{23,24}$ The B term is derived from the coupling between the intermediate electronic excited state to other excited states, while the $\mathrm{C}$ term is due to the coupling between the ground electronic state to excited states and is customarily assumed to be small. The D term is of higher order in the coupling between electronic states and is often neglected. Albrecht's treatment involves a full sum over all vibronic states of the molecule and is thus rarely computationally tractable for larger systems. Nevertheless, it constituted a major breakthrough in the understanding of Raman scattering in that it provided a unified picture for both non-resonant and resonant Raman spectra. Sums over vibronic states can be evaluated in the displaced harmonic oscillator approximation. ${ }^{25}$

A different approach to resonance Raman scattering was proposed by Heller and co-workers. ${ }^{26}$ It amounts to a transformation of the KHD dispersion relation into the time domain, which represents the resonance Raman process as a propagation of vibrational wavepackets (multiplied with transition dipole moments) on the excited-state potential energy surface. Often, the short-time approximation to propagation dynamics is introduced, ${ }^{27}$ which has proven remarkably useful in interpreting resonance Raman spectra. ${ }^{27-29}$

Finally, resonance Raman cross section can be expressed in a fashion analogous to the nonresonant case by introducing finite lifetimes for the intermediate states, or in other words, by computing Raman cross sections from derivatives of electronic polarizabilities evaluated at complex frequencies $\tilde{\omega}=\omega+i \gamma \cdot{ }^{30,31}$ Here $\omega$ is the excitation frequency and $\gamma$ corresponds to an averaged lifetime of excited states, which is usually treated as an empirical parameter.

The purpose of the present work is to provide a simple and computationally tractable approximation for resonance Raman cross sections. To this end, we reduce the summation over vibronic states of the KHD dispersion relation to a summation of electronic states, similar to the parametric method of Shorygin and co-workers, and apply the double harmonic approximation, which is commonly used in calculations of vibrational spectra. This approximation requires only excitation 
energies, transition dipole moments, and their respective geometric derivatives to be computed for the electronic excited states included in the sum-over-states expression. In contrast to Shorygin's work, all parameters in the sum-over-states expression are provided from ab initio calculations, while the summation runs over all electronic excitations in a given energy range. Analytical gradient techniques make computation of geometric derivatives particularly efficient in the framework of time-dependent density functional theory (TDDFT). ${ }^{32}$ In addition, the sum-over-states approach may be used to identify major contributions to resonance Raman intensities. We apply the present approach to assign and interpret resonance Raman scattering in nucleic acid bases.

The polarizability theory of Raman scattering due to Placzek relates the Raman scattering cross section to frequency-dependent electronic polarizabilities at the frequency of the incident radiation, ${ }^{17,21}$

$$
\alpha^{m n}(\omega)=\sum_{k}\left[\frac{\mu_{0 k}^{m} \mu_{0 k}^{n}}{\Omega_{k}-\omega}+\frac{\mu_{0 k}^{n} \mu_{0 k}^{m}}{\Omega_{k}+\omega}\right] .
$$

$m$ and $n$ are Cartesian directions. We use atomic units throughout. The summation is over all electronic excited states $k>0$ with excitation energies $\Omega_{k}$ and transition dipole moments $\mu_{0 k}^{m}$. The polarizability theory of Raman scattering is based on the separability of the electronic and nuclear wavefunctions (Born-Oppenheimer approximation) and the assumption that the incident radiation is sufficiently far from resonance such that energy differences between vibronic levels of the KHD expression may be approximated by electronic excitation energies $\Omega_{k}$. In the double harmonic approximation, the Raman scattering cross sections are proportional to derivatives of $\alpha^{m n}(\omega)$ with respect to vibrational normal modes. ${ }^{17,21}$ Straightforward differentiation of the sum-over-states expansion for $\alpha(\omega)$ with respect to the vibrational normal mode $Q$ yields the following expression for the Raman scattering cross section of the vibration $Q$,

$$
\left(\frac{\partial \sigma}{\partial \Omega}\right)_{Q}=\frac{\left(\omega-\omega_{Q}\right)^{4}}{2 \omega_{Q} c^{4}}\left|\left\langle\boldsymbol{\sigma}_{Q}(\omega)\right\rangle\right|^{2}
$$


where the components of the Raman scattering tensor $\sigma_{Q}^{m n}(\omega)$ are given by

$$
\begin{aligned}
\sigma_{Q}^{m n}(\omega)= & \sum_{k}\left[-\mu_{0 k}^{m} \mu_{0 k}^{n}\left[\frac{\left(\Omega_{k}-\omega\right)^{2}-\gamma_{k}^{2}}{\left(\left(\Omega_{k}-\omega\right)^{2}+\gamma_{k}^{2}\right)^{2}}+\frac{i 2\left(\Omega_{k}-\omega\right) \gamma_{k}}{\left(\left(\Omega_{k}-\omega\right)^{2}+\gamma_{k}^{2}\right)^{2}}\right] \frac{\partial \Omega_{k}}{\partial Q}\right. \\
& \left.+\left[\mu_{0 k}^{m} \frac{\partial \mu_{0 k}^{n}}{\partial Q}+\frac{\partial \mu_{0 k}^{m}}{\partial Q} \mu_{0 k}^{n}\right]\left[\frac{\Omega_{k}-\omega}{\left(\Omega_{k}-\omega\right)^{2}+\gamma_{k}^{2}}+\frac{i \gamma_{k}}{\left(\Omega_{k}-\omega\right)^{2}+\gamma_{k}^{2}}\right]\right]
\end{aligned}
$$

Here, $\omega_{Q}$ is the vibrational frequency, $c$ is the speed of light. Angle brackets denote the appropriate orientational average over components of the Raman scattering tensor $\boldsymbol{\sigma}_{Q}(\omega)$. The excited states $k>0$ have linewidths $\gamma_{k}$ associated with them, which are chosen as empirical parameters independent of $k$ in most studies. We will follow this practice here. The analogous expression for $\sigma_{Q}^{m n}(\omega)$ with uniform linewidths $\gamma_{k}=\gamma$ for all excited states may be obtained by differentiation of the polarizability evaluated at the complex frequency $\tilde{\omega}=\omega+i \gamma \cdot{ }^{30,31}$ In contrast, in the present approach different linewidths $\gamma_{k}$ may be chosen for individual excited states to reflect differences in their lifetimes. Ultimately, the excited-state linewidths may be rigorously derived from a open-system formulation, e. g., in the framework of TDDFT. ${ }^{33-35}$

In practice, the sum over electronic excited states has to be truncated. The number of excited states contributing significantly to the Raman cross sections in Eq. (3) will be small in the vicinity of a resonance $\left(\left|\omega-\Omega_{k}\right| \approx \gamma_{k}\right)$ but might increase significantly in the non-resonant case. While truncation of the sum-over-states is a potential source of error not present in the finite-lifetime approach, ${ }^{30,31}$ we find that convergence is sufficiently fast even in the non-resonant regime for nucleic acid bases considered here.

Differentiation of the frequency-dependent electronic polarizabilities (Eq. (1)) with respect to the vibrational normal mode $Q$ gives rise to two kinds of terms for each excited state. The first term in Eq. (3) is proportional to the Cartesian derivative (gradient) of the excitation energy $\frac{\partial \Omega_{k}}{\partial Q}$. It may be compared to the A term in Albrecht's approach, which arises from the energy differences between vibronic states in the energy denominator. ${ }^{16,17}$ By analogy, we will refer to these contributions as the A terms in the following. Only totally symmetric vibrational modes $Q$ yield non-zero energy derivatives $\frac{\partial \Omega_{k}}{\partial Q}$, therefore A terms are only present for totally symmetric vibrations. The 
second term in Eq. (3) results from the dependence of transition moments $\mu_{0 k}^{m}$ on the vibrational normal modes. In the language of Herzberg-Teller coupling, ${ }^{16,23,24}$ this dependence results from the interaction of the ground state or the electronic excited state $k$ with other electronic states induced by nuclear displacements along the vibrational mode $Q$. The corresponding contributions are denoted B and C terms, respectively, in Albrecht's approach. The terms in Eq. (3) that are proportional to derivatives of transition dipole moments $\frac{\partial \mu_{0 k}^{m}}{\partial Q}$ have the same origin and hence will be referred to as B terms. B terms are non-zero for vibrational modes that transform like components of the polarizability tensor; the selection rules for the B term are equivalent to those for non-resonant Raman scattering. ${ }^{16,17}$

The frequency dependence of Raman spectra is defined by the molecular electronic excitation spectrum. In the strictly resonant case $\left(\omega=\Omega_{k}\right)$ the excited electronic state $k$ dominates the sum in Eq. (3). In this limit, the shape of the resonance Raman spectrum reflects the structure of the potential energy surface of the excited state $k$. Since the A term is quadratic in the resonance denominator $\left(\left(\Omega_{k}-\omega\right)^{2}+\gamma_{k}^{2}\right)^{-1}$, while the B term is linear in it, the A term contribution can be expected to be predominant at resonance. In the opposite limiting case the excitation frequency is far from any electronic excitations (non-resonant Raman scattering), and a considerable number of electronic excited states contributes to the sum-over-states expression, Eq. (3). The B term contributions become dominant in Raman cross sections, while the A terms are scaled down by their large energy denominators. Smooth interpolation between both limiting cases (non-resonant and strictly resonant) requires that both $\mathrm{A}$ and $\mathrm{B}$ terms be treated on equal footing.

Analytical derivative techniques allow to compute excitation energy gradients and non-resonant polarizability derivatives in an efficient fashion using TDDFT. ${ }^{32,36}$ In this work, derivatives of transition dipole moments are computed by numerical differentiation. However, an analytical implementation is possible starting from a Lagrangian formulation, ${ }^{37}$ similar to that for gradients of excitation energies $^{38,39}$ and frequency-dependent polarizabilities. ${ }^{36}$

In the following, we explore the characteristic changes in resonance Raman spectra of guanosine for excitations in the range between $200-266 \mathrm{~nm}$, which contains a number of electronic 
excitations. In addition, we consider Raman excitation profiles of ring-breathing modes of nucleosides. Raman excitation profiles describe the dependence of Raman cross sections on the energy of the incident radiation. Finally, we determine the relative contributions of the A and B terms to Raman cross sections of guanosine both at resonance and in the non-resonant case.

All calculations have been performed using the PBE0 functional ${ }^{40}$ and triple-zeta valence basis sets with two sets of polarization functions (TZVPP). ${ }^{41}$ The PBE0 functional has been chosen because it has proven quite accurate both for polarizabilities ${ }^{42,43}$ and Raman intensities. ${ }^{36,44}$ However, vibrational frequencies ${ }^{45}$ and electronic excitation energies ${ }^{46}$ are often overestimated with PBE0. 20 excited electronic states were included in the sum-over-states expressions. Linewidth parameters were assumed to be $0.1 \mathrm{eV}$ for all electronic states. All calculations were performed using the program package TURBOMOLE. ${ }^{47}$

In Figure 1(a)-(c), we compare experimental and computed resonance Raman spectra of guanosine at excitation wavelengths of $266 \mathrm{~nm}, 218 \mathrm{~nm}$, and $200 \mathrm{~nm}$. In addition, we show experimental and computed non-resonant Raman spectra of guanosine at $514.5 \mathrm{~nm}$ in Figure 1(d). The experimental spectra are from Refs. ${ }^{48,49}$ The considered range of excitation energies includes the two overlapping electronic absorption bands of guanosine observed experimentally at 4.4-4.6 eV and $4.8-5.1 \mathrm{eV} .{ }^{50-52}$ Deconvolution of the UV absorption spectrum of guanosine in water yields 4.56 $\mathrm{eV}$ and $5.04 \mathrm{eV}$ for the positions of the absorption maxima. ${ }^{50} \mathrm{PBE} 0$ predicts the two lowest electronic excited states of guanosine at $4.97 \mathrm{eV}$ and $5.39 \mathrm{eV}$ to be strongly allowed. At still higher excitation energies, a second pair of strongly allowed electronic absorption bands is observed experimentally, ${ }^{50,52}$ with maxima at $6.17 \mathrm{eV}$ and $6.67 \mathrm{eV}$, respectively. The computed excitation energies for these transitions are $6.79 \mathrm{eV}$ and $6.99 \mathrm{eV}$. We refer to supplementary information for a full overview of computed and experimental excitation energies of guanosine. The overestimation of excitation energies observed here is quite typical for the PBE0 functional ${ }^{46}$ and is in part due to the lack of solvation effects in the calculations. Since the shape of resonance Raman spectra is sensitive to the relative position of the frequency of the incident light in the electronic excitation spectrum, we correct for the systematic error in excitation energies with PBE0. To this end, we first 

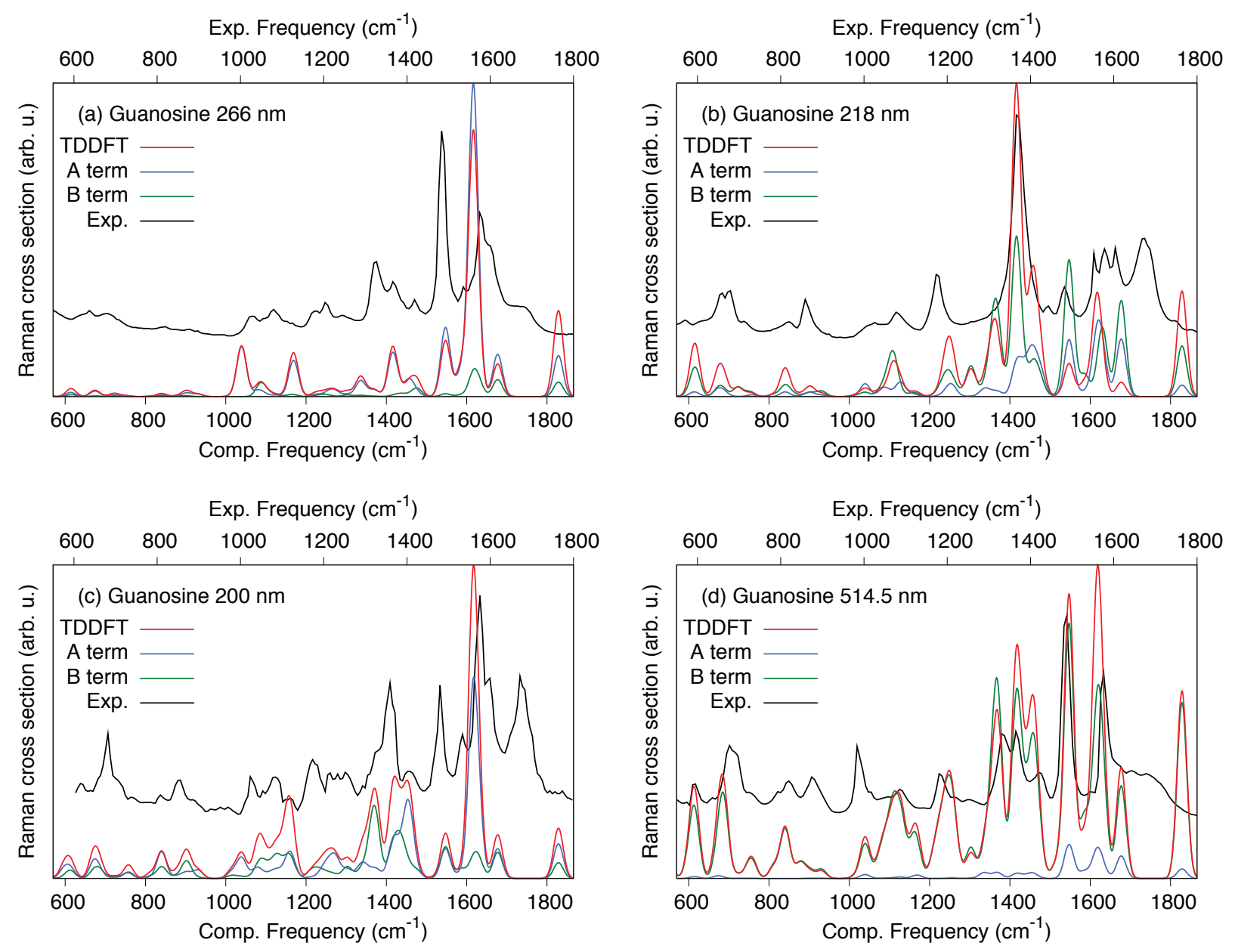

Figure 1: Experimental and computed Raman spectra of guanosine at 266, 218, 200, and 514.5 $\mathrm{nm}$ excitations. Experimental spectra of guanosine- $5^{\prime}$-monophosphate (GMP) are from Refs. ${ }^{48,49}$ Note that different frequency scales are applied to experimental and computed Raman spectra. See text for computational details. 
introduce a linear regression between the computed and experimental excitation energies based on the four strongly allowed electronic transitions of guanosine. The slope of the linear regression is 1.02 , the offset is $0.35 \mathrm{eV}$. In addition, frequency scales in experimental and computed Raman spectra are adjusted in Figure 1(a)-(d) to reflect the systematic overestimation of vibrational frequencies with PBE0 functional. ${ }^{45}$ This corresponds to an effective scaling factor of 0.96 .

The experimental resonance Raman spectrum at $266 \mathrm{~nm}$ excitation (Figure 1(a)) is characterized by a strong $1492 \mathrm{~cm}^{-1}$ Raman peak and a slightly less intense $1581 \mathrm{~cm}^{-1}$ band. The former vibrational band was attributed to an imidazole ring vibration while the latter was assigned to a pyrimidine ring stretch mode. ${ }^{53}$ A complete assignment of intensive Raman bands of guanosine is given in the supplementary information. To facilitate comparison between experimental and theoretical results, we compute the resonance Raman spectra at an excitation frequency shifted according to the linear regression results, see above. The experimental results obtained using 266 $\mathrm{nm}(4.66 \mathrm{eV})$ excitation are thus compared to computed Raman spectra at the $245 \mathrm{~nm}(5.07 \mathrm{eV})$ excitation. The computed Raman spectrum at $245 \mathrm{~nm}$ is dominated by contributions from the $S_{1}$ excited state. The strongest vibrational band is found at $1631 \mathrm{~cm}^{-1}$ and stems from the $v\left(\mathrm{~N}_{7}-\mathrm{C}_{8}\right)$ bond stretch. The pyrimidine ring stretch is observed as a weaker band at $1547 \mathrm{~cm}^{-1}$. Comparison with the resonance Raman spectrum computed for the $S_{2}$ electronic excitation shows the opposite pattern, with a strong band at $1547 \mathrm{~cm}^{-1}$ and a somewhat less intense one at $1631 \mathrm{~cm}^{-1}$. The predicted spectrum at resonance with the $S_{2}$ state seems to be in a better overall agreement with the experimental resonance Raman spectrum at $266 \mathrm{~nm}$ than the computed spectrum at resonance with the $S_{1}$ state, see supplementary information for more details. This findings underscore the importance of an accurate determination of the relative position of the frequency of the incident radiation relative to the electronic excitation spectrum of the molecule. The linear regression between experimental and computed excitations used here is perhaps the simplest possible correction scheme, while more rigorous approaches would contributions from the A terms, as is expected for an excitation close to resonance.

The experimental resonance Raman spectrum for the $218 \mathrm{~nm}$ excitation is characterized by a 
strong vibrational band at $1367 \mathrm{~cm}^{-1}$ assigned to an in-plane purine ring vibration. The $v\left(\mathrm{C}_{6}=\mathrm{O}\right)$ Raman band is observed at $1685 \mathrm{~cm}^{-1}$. The corresponding computed spectrum is obtained for the $203 \mathrm{~nm}(6.11 \mathrm{eV})$ excitation. The intermediate $\pi \rightarrow \pi^{*}$ excited state $S_{10}$ of guanosine of low intensity (computed excitation energy $6.25 \mathrm{eV}$ ) has the largest contribution to the computed resonance Raman spectrum. It might be associated with the electronic transition observed at 215 $\mathrm{nm}(5.77 \mathrm{eV})$ in circular dichroism (CD) spectra of guanosine. ${ }^{54}$ Due to the low oscillator strength of the $S_{10}$ transition (0.05), the resonance Raman intensity is derived from both the A and the B terms. The strongest vibrational band in the computed resonance Raman spectrum at $203 \mathrm{~nm}$ excitation purine ring stretch mode predicted at $1416 \mathrm{~cm}^{-1}$.

The experimental resonance Raman spectrum at $200 \mathrm{~nm}$ shows a strong pyrimidine ring stretching band at $1578 \mathrm{~cm}^{-1}$ vibrational band as well as three Raman peaks of nearly equal intensity at $1679 \mathrm{~cm}^{-1}, 1489 \mathrm{~cm}^{-1}$, and $1364 \mathrm{~cm}^{-1}$, which are assigned to the $v\left(\mathrm{C}_{6}=\mathrm{O}\right)$ stretch, a pyrimidine ring stretch, and an imidazole ring stretch, respectively. The low-frequency part of the resonance Raman spectrum is dominated by the ring breathing mode. The computed resonance Raman spectrum at $187 \mathrm{~nm}(6.63 \mathrm{eV})$ is close in energy to the strongly allowed $\pi \rightarrow \pi^{*}$ state $\left(S_{13}\right)$ at $6.79 \mathrm{eV}$. The pyrimidine ring stretch vibration at $1631 \mathrm{~cm}^{-1}$ is predicted as the strongest vibrational band. The intensities of the the $v\left(\mathrm{C}_{6}=\mathrm{O}\right)$ vibration at $1829 \mathrm{~cm}^{-1}$, the imidazole ring vibration at 1547 $\mathrm{cm}^{-1}$, and the ring deformation mode at $1416 \mathrm{~cm}^{-1}$, which correspond to the three intense Raman bands observed experimentally, are underestimated relative to the strongest Raman peak. Since the excitation at $200 \mathrm{~nm}$ is close to strict resonance, the A terms are dominant in the resonance Raman spectrum.

The non-resonant Raman spectrum of guanosine at $514.5 \mathrm{~nm}$ is shown in Figure 1(d). Assignments of the non-resonant Raman spectra of guanine and its derivatives have been published previously. ${ }^{55-57}$ As expected for Raman spectra far from resonance, the B terms are dominant, while the A terms are comparatively small. The non-resonant case is characterized by a significant number of excited electronic states, each contributing only a small amount to Raman cross sections. Under these circumstances, the closure of the sum over states is applicable, and the resulting 
Raman cross sections are represented as a ground state response property. ${ }^{17,21}$ The sum-over-states results for guanosine Raman spectra at $514.5 \mathrm{~nm}$ including 20 excited electronic states is in very good agreement with the conventional result obtained from derivatives of frequency-dependent electronic polarizabilities, see supplementary information.

The changes observed in the experimental resonance Raman spectra can be well described within the sum-over-states formalism. A comprehensive assignment of Raman peaks can be achieved. The relative changes in resonance Raman spectra depend on a the relative position of the frequency of the incident radiation within the electronic excitation spectrum. Thus a balanced description of a large number of electronic excitations is required, which represents a considerable challenge for the existing DFT methodology. Generally, the sum-over-states approach reproduces the characteristic changes in the overall shape of resonance Raman spectra reasonably well. This suggests that the main source of error in these calculations is due to electronic excitation energies, while the local properties of excited states, such as energy gradients and derivatives of transition dipole moments, are better reproduced. Similar results have been found for relaxed structures of excited states. ${ }^{32,38,39}$ However, we note that all comparisons include relative Raman cross sections only. Accurate determination of absolute Raman cross sections is a challenging taks both for experiments and computation and is not considered here.

Raman excitation profiles (REPs) describe the dependence of Raman scattering cross sections on excitation frequency. In Figure 2 we show the REPs for the ring breathing modes of adenosine, guanosine, cytidine, and uridine. These low-frequency totally symmetric vibrational modes correspond to an in-phase expansion or contraction of the entire heteroaromatic ring system. Experimental spectra are from Ref. ${ }^{58}$ For consistency, the correction for systematic errors in excitation energies derived for guanosine (see above for details) is used for all nucleosides.

The ring breathing mode of adenosine (Figure 2(a)) is observed at $729 \mathrm{~cm}^{-1}$ in experimental spectra, while the computed vibrational frequency is $747 \mathrm{~cm}^{-1}$. The experimental REP shows two maxima at the positions of the electronic absorption bands of adenosine. They are assigned to the strongly allowed $\pi \rightarrow \pi^{*}$ excited states $S_{2}$ and $S_{7}$, respectively. The Raman cross section of the 
Exp. Excitation Energy (eV)

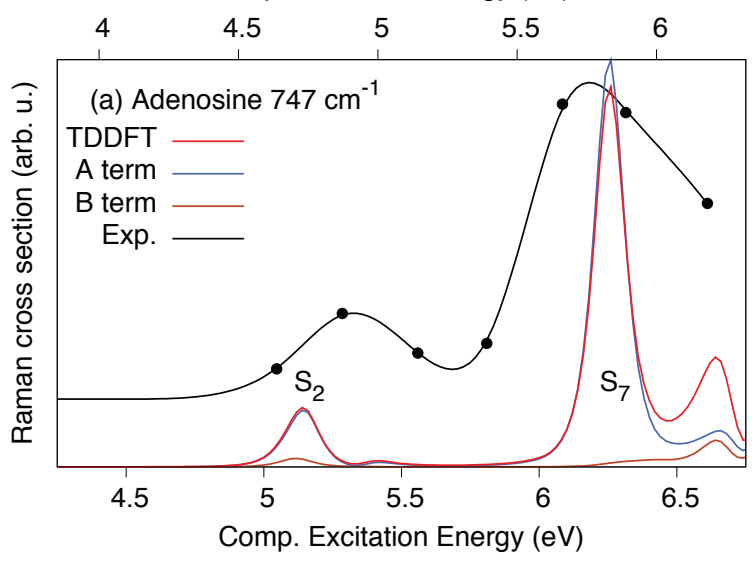

Exp. Excitation Energy (eV)

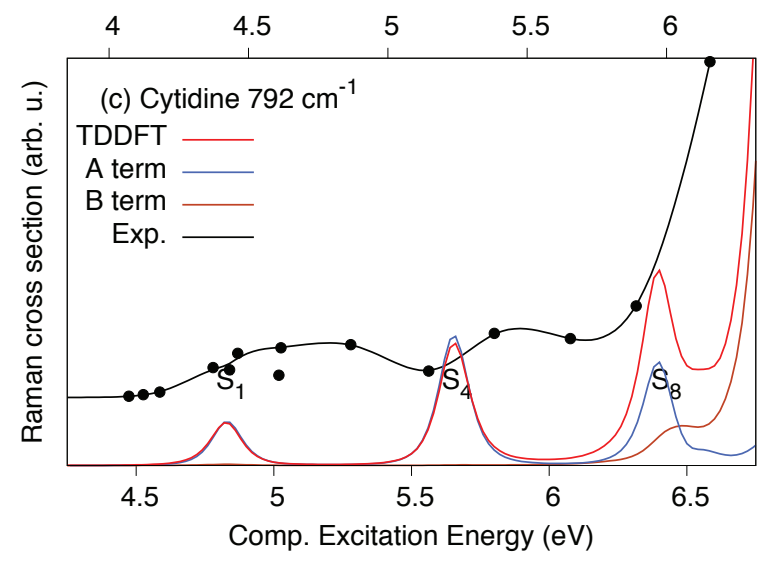

Exp. Excitation Energy (eV)

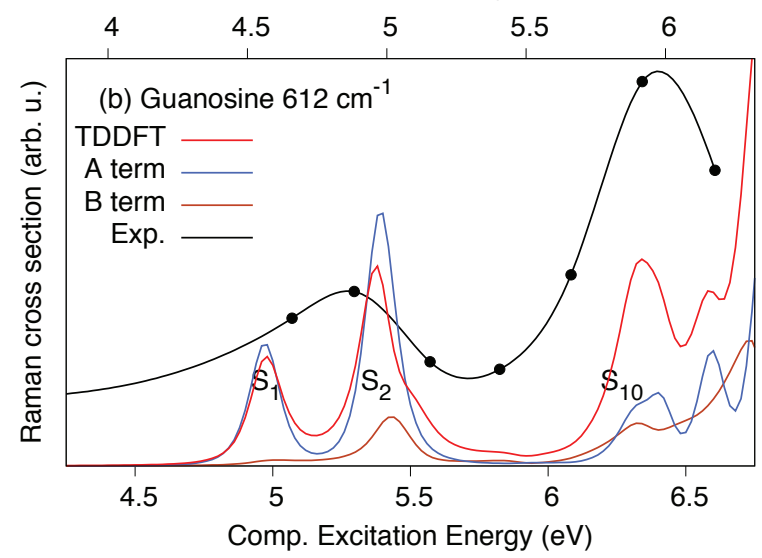

Exp. Excitation Energy (eV)

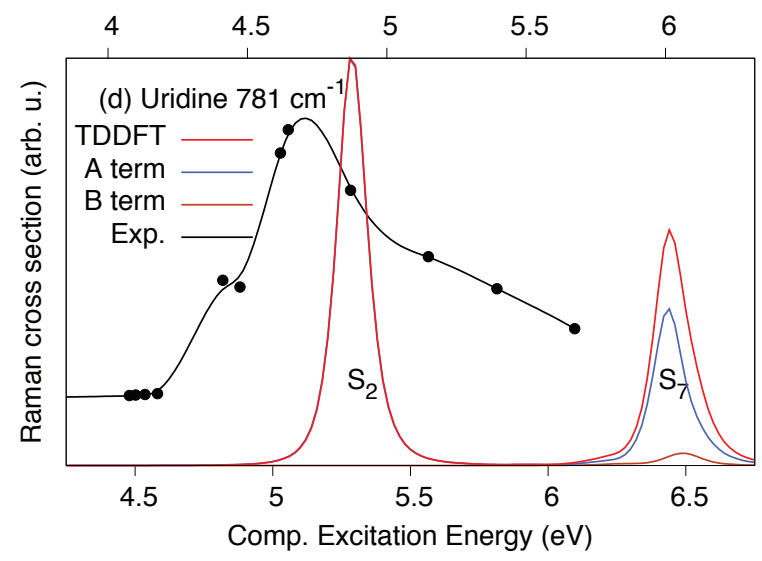

Figure 2: Experimental and computed Raman excitation profiles of ring breathing modes of nucleosides. Experimental data for nucleoside $5^{\prime}$-monophosphates are from Ref. ${ }^{58}$ Solid lines in experimental data are obtained by interpolation and serve solely to guide the eye. Note that different energy scales are applied to experimental and computed Raman excitation profiles. See text for computational details. 
ring breathing mode is larger at resonance with the higher-energy absorption band, in line with experimental data. Since the ring breathing vibration is totally symmetric, its intensity is almost entirely due to A term contributions.

The ring breathing mode of guanosine (Figure 2(b)) is observed at $670 \mathrm{~cm}^{-1}$; the computed vibrational frequency is $612 \mathrm{~cm}^{-1}$. Two broad maxima are observed in the experimental REP at the positions of the two electronic absorption bands. The first REP maximum at 4.5-5.0 eV covers the two closely lying dipole-allowed states $S_{1}$ and $S_{2}$, while the second REP maximum peaked at ca. $6 \mathrm{eV}$ includes the weakly allowed $S_{10}$ state as well as the strongly absorbing $S_{13}$ and $S_{17}$ states. The larger Raman cross section at the second maximum is reproduced by theoretical results. The significant contribution from B terms, which grows with increasing excitation energy, suggests that the ring breathing modes is strongly coupled to non-totally symmetric vibrations.

Experimental and computed REPs of the ring breathing mode of cytidine are shown in Figure 2(c). Experimental vibrational frequency is $782 \mathrm{~cm}^{-1}$, the computed frequency is $792 \mathrm{~cm}^{-1}$. Two moderately strong maxima are present in the experimental REP, followed by a significant increase at the high-energy edge of the REP. The two maxima are attributed to the two $\pi \rightarrow \pi^{*}$ transitions of cytidine $\left(S_{1}\right.$ and $\left.S_{4}\right)$. The increase at above $6.2 \mathrm{eV}$ is due to the $S_{8}$ and higher-lying excited states. While the Raman cross sections are almost exclusively due to A terms at the first maxima, the contribution of B terms increases at higher excitation energies. The ring breathing mode of uridine shows a single broad peak at about $4.7 \mathrm{eV}$ (Figure 2(d)), which is assigned to the strongly allowed $S_{2}$ excited state. Experimental vibrational frequency of the ring breathing mode is $783 \mathrm{~cm}^{-1}$, computed value is $781 \mathrm{~cm}^{-1}$.

The two limiting cases of the sum-over-states expression are the strictly resonant situation, in which one resonant electronic state dominates the sum, with the A terms outweighing the corresponding B terms. In this case, the sum reduces to the short-time approximation. ${ }^{59}$ The other limiting case, far from resonance, is usually well described by the polarizability theory of Placzek, ${ }^{21}$ in which polarizability derivatives are often even approximated by their static limits. As was pointed out by Albrecht, B terms are dominant in the non-resonant case, ${ }^{16}$ while A terms are all but negli- 
gible. The polarizability approximation is usually adequate for the range of excitation frequencies below the lowest electronic excitation. In the intermediate regime, e. g., above the first electronic excitation, both A and B terms from different electronic excited states contribute to Raman cross section, and a smooth interpolation, such as the one offered by the present approach, becomes necessary.

The presented sum-over-states approach ignores the details of vibronic structure and includes the contributions from a given electronic excited state in an aggregate manner only. Thus, it is likely to be problematic for molecules with well-resolved vibronic transitions such as small gasphase species. However, vibronic structure is typically "washed out" in most medium-size and large molecules or in the presence of a solvent so that the averaged description appears appropriate in these cases. The contributions of different electronic excited states to the Raman scattering tensor $\sigma_{Q}(\omega)$ are additive, cf. Eq. (2). Therefore, the quality of the description of the strictly resonant case may be improved upon by treating the contribution of the resonant electronic state in a more accurate way such as explicit time propagation ${ }^{26,27}$ or summation over vibronic states. ${ }^{25}$

The computed resonance Raman cross sections include excitation energies, transition moments and their geometric derivatives. As a consequence, they offer a sensitive test of the TDDFT methodology. Our results indicate that largest source of error for relative resonance Raman cross sections are excitation energies, and the results are found to improve if the excitation energies are corrected for errors intrinsic to the method. Corrections using experimental excitation energies might be used for this purpose if available. Alternatively, corrections for excitation energies might be obtained from more accurate theoretical methods such as coupled-cluster response approaches.

In this work, we presented a simple approximation to resonance Raman cross section based on the sum-over-states expression for frequency-dependent electronic polarizabilities. Each electronic excited state contributes two types of terms to the Raman cross section, which we term A and B terms, in analogy to Albrecht's treatment. The A terms are dominant in the strictly resonant case, while the B terms determine the Raman cross sections in the non-resonant limit. By using both terms, the present method can treat the resonant and non-resonant cases on equal footing. 
Resonance Raman spectra and Raman excitation profiles of nucleosides can be predicted with reasonable accuracy using the sum-over-states approach. The major source of error seem to be electronic excitation energies, which are can be off by up to $0.5 \mathrm{eV}$ with TDDFT. Improved description of resonance Raman spectra and Raman excitation profiles is expected from a combination of the present sum-over-states formulation with more accurate approaches for the few strictly resonant electronic states as well as an first-principles framework for computing electronic state linewidths from the open-system formulation of TDDFT. ${ }^{35}$

\section{Acknowledgement}

The authors acknowledge support from DARPA under contract no. FA9550-08-1-0285. D. R. acknowledges the Postdoctoral Research Award presented by the Physical Chemistry Division of the American Chemical Society and the Journal of Physical Chemistry Letters.

Supporting Information Available: Assignment of Raman-active vibrational modes of guanosine, computed and experimental electronic excitations of DNA nuclear acid bases and nucleosides, computed strictly resonant and non-resonant Raman spectra of guanosine. This material is available free of charge via the Internet at http://pubs.acs.org.

\section{References}

(1) Shorygin, P. P. Intensity of Combination Scattering Lines and Structure of Organic Compounds. Zh. Fiz. Khim. 1947, 21, 1125-1134.

(2) Shorygin, P. P.; Krushinskij, L. L. Early Days and Later Development of Resonance Raman Spectroscopy. J. Raman Spectrosc. 1997, 28, 383-388.

(3) Parker, F. S. Applications of Infrared, Raman, and Resonance Raman Spectroscopy in Biochemistry; Springer: New York, 1983.

(4) Spiro, T. G., Ed. Biological Applications of Raman Spectroscopy; Wiley: New York, 1987; Vol. 1-3. 
(5) Benevides, J. M.; Overman, S. A.; Thomas, G. J. Raman, Polarized Raman and Ultraviolet Resonance Raman Spectroscopy of Nucleic Acids and Their Complexes. J. Raman Spectrosc. 2005, 36, 279-299.

(6) Moskovits, M. Surface-Enhanced Spectroscopy. Rev. Mod. Phys. 1985, 57, 783-826.

(7) Kneipp, K., Moskovits, M., Kneipp, H., Eds. Surface-Enhanced Raman Scattering: Physics and Applications; Springer: Berlin, 2006.

(8) Jensen, L.; Aikens, C. M.; Schatz, G. C. Electronic Structure Methods for Studying SurfaceEnhanced Raman Scattering. Chem. Soc. Rev. 2008, 37, 1061-1073.

(9) Schatz, G.; Young, M.; Van Duyne, R. In Surface-Enhanced Raman Scattering; Kneipp, K., Moskovits, M., Kneipp, H., Eds.; Springer: Berlin, 2006; Vol. 103; pp 19-45.

(10) Saikin, S. K.; Olivares-Amaya, R.; Rappoport, D.; Stopa, M.; Aspuru-Guzik, A. On the Chemical Bonding Effects in the Raman Response: Benzenethiol Adsorbed on Silver Clusters. Phys. Chem. Chem. Phys. 2009, 11, 9401-9411.

(11) Saikin, S. K.; Chu, Y.; Rappoport, D.; Crozier, K. B.; Aspuru-Guzik, A. Separation of Electromagnetic and Chemical Contributions to Surface-Enhanced Raman Spectra on Nanoengineered Plasmonic Substrates. J. Phys. Chem. Lett. 2010, 2740-2746.

(12) Nie, S.; Emory, S. R. Probing Single Molecules and Single Nanoparticles by SurfaceEnhanced Raman Scattering. Science 1997, 275, 1102.

(13) Kneipp, K.; Wang, Y.; Kneipp, H.; Perelman, L. T.; Itzkan, I.; Dasari, R. R.; Feld, M. S. Single Molecule Detection Using Surface-Enhanced Raman Scattering (SERS). Phys. Rev. Lett. 1997, 78, 1667-1670.

(14) Dieringer, J. A.; Wustholz, K. L.; Masiello, D. J.; Camden, J. P.; Kleinman, S. L.; Schatz, G. C.; Van Duyne, R. P. Surface-Enhanced Raman Excitation Spectroscopy of a Single Rhodamine 6G Molecule. J. Am. Chem. Soc. 2009, 131, 849-854. 
(15) Schorygin, P.; Kuzina, L.; Ositjanskaja, L. Untersuchungen über die Intensität der Ramanlinien. Microchim. Acta 1955, 43, 630-636.

(16) Albrecht, A. C. On the Theory of Raman Intensities. J. Chem. Phys. 1961, 34, 1476-1484.

(17) Long, D. A. The Raman Effect, 2nd ed.; Wiley: Chichester, 2002.

(18) Kramers, H. A.; Heisenberg, W. Über die Streuung von Strahlung durch Atome. Z. Phys. 1925, 31, 681-708.

(19) Dirac, P. A. M. The Quantum Theory of Dispersion. Proc. Roy. Soc. A 1927, 114, 710-728.

(20) van Vleck, J. H. On the Vibrational Selection Principles in the Raman Effect. Proc. Nat. Acad. Sci. 1929, 15, 754-764.

(21) Placzek, G. In Handbuch der Radiologie; Marx, E., Ed.; Akademische Verlagsgesellschaft: Leipzig, 1934; Vol. VI/2; pp 209-374.

(22) Behringer, J.; Brandmüller, J. Der Resonanz-Raman-Effekt. Z. Elektrochem. 1956, 60, 643679.

(23) Albrecht, A. C. "Forbidden" Character in Allowed Electronic Transitions. J. Chem. Phys. 1960, 33, 156-169.

(24) Fischer, G. Vibronic Coupling: the Interaction between the Electronic and Nuclear Motions; Academic Press: London, 1984.

(25) Kane, K. A.; Jensen, L. Calculation of Absolute Resonance Raman Intensities: Vibronic Theory vs Short-Time Approximation. J. Phys. Chem. C 2010, 114, 5540-5546.

(26) Lee, S.-Y.; Heller, E. J. Time-Dependent Theory of Raman Scattering. J. Chem. Phys. 1979, $71,4777-4788$.

(27) Tannor, D. J. Polyatomic Raman Scattering for General Harmonic Potentials. J. Chem. Phys. 1982, 77, 202. 
(28) Neugebauer, J.; Hess, B. A. Resonance Raman Spectra of Uracil Based on Kramers-Kronig Relations Using Time-Dependent Density Functional Calculations and Multireference Perturbation Theory. J. Chem. Phys. 2004, 120, 11564-11577.

(29) Neese, F.; Petrenko, T.; Ganyushin, D.; Olbrich, G. Advanced Aspects of Ab Initio Theoretical Optical Spectroscopy of Transition Metal Complexes: Multiplets, Spin-Orbit Coupling and Resonance Raman Intensities. Coord. Chem. Rev. 2007, 251, 288-327.

(30) Jensen, L.; Autschbach, J.; Schatz, G. C. Finite Lifetime Effects on the Polarizability Within Time-Dependent Density-Functional Theory. J. Chem. Phys. 2005, 122, 224115.

(31) Jensen, L.; Zhao, L. L.; Autschbach, J.; Schatz, G. C. Theory and Method for Calculating Resonance Raman Scattering from Resonance Polarizability Derivatives. J. Chem. Phys. 2005, $123,174110$.

(32) Furche, F.; Rappoport, D. In Computational Photochemistry; Olivucci, M., Ed.; Theoretical and Computational Chemistry; Elsevier: Amsterdam, 2005; pp 93-128.

(33) Yuen-Zhou, J.; Tempel, D. G.; Rodríguez-Rosario, C. A.; Aspuru-Guzik, A. Time-Dependent Density Functional Theory for Open Quantum Systems with Unitary Propagation. Phys. Rev. Lett. 2010, 104, 043001.

(34) Yuen-Zhou, J.; Rodríguez-Rosario, C. A.; Aspuru-Guzik, A. Time-Dependent CurrentDensity Functional Theory for Generalized Open Quantum Systems. Phys. Chem. Chem. Phys. 2009, 11, 4509-4522.

(35) Tempel, D. G.; Watson, M. A.; Olivares-Amaya, R.; Aspuru-Guzik, A. Time-Dependent Density Functional Theory of Open Quantum Systems in the Linear-Response Regime. J. Chem. Phys. 2011, 134, 074116.

(36) Rappoport, D.; Furche, F. Lagrangian Approach to Molecular Vibrational Raman Intensi- 
ties Using Time-Dependent Hybrid Density Functional Theory. J. Chem. Phys. 2007, 126, 201104.

(37) Coriani, S.; Kjærgaard, T.; Jørgensen, P.; Ruud, K.; Huh, J.; Berger, R. An Atomic-OrbitalBased Lagrangian Approach for Calculating Geometric Gradients of Linear Response Properties. J. Chem. Theory Comput. 2010, 6, 1028-1047.

(38) Furche, F.; Ahlrichs, R. Adiabatic Time-Dependent Density Functional Methods for Excited State Properties. J. Chem. Phys. 2002, 117, 7433-7447.

(39) Rappoport, D.; Furche, F. In Time-Dependent Density Functional Theory; Marques, M. A. L., Ullrich, C. A., Nogueira, F., Rubio, A., Burke, K., Gross, E. K. U., Eds.; Springer: Berlin, Chapter 23, pp 337-354.

(40) Perdew, J. P.; Ernzerhof, M.; Burke, K. Rationale for Mixing Exact Exchange With Density Functional Approximations. J. Chem. Phys. 1996, 105, 9982-9985.

(41) Schäfer, A.; Huber, C.; Ahlrichs, R. Fully Optimized Contracted Gaussian Basis Sets of Triple Zeta Valence Quality for Atoms Li to Kr. J. Chem. Phys. 1994, 100, 5829-5835.

(42) Adamo, C.; Cossi, M.; Scalmani, G.; Barone, V. Accurate Static Polarizabilities by Density Functional Theory: Assessment of the PBE0 Model. Chem. Phys. Lett. 1999, 307, 265-271.

(43) Van Caillie, C.; Amos, R. D. Static and Dynamic Polarisabilities, Cauchy Coefficients and Their Anisotropies: an Evaluation of DFT Functionals. Chem. Phys. Lett. 2000, 328, 446452.

(44) Van Caillie, C.; Amos, R. D. Raman Intensities Using Time Dependent Density Functional Theory. Phys. Chem. Chem. Phys. 2000, 2, 2123-2129.

(45) Merrick, J. P.; Moran, D.; Radom, L. An Evaluation of Harmonic Vibrational Frequency Scale Factors. J. Phys. Chem. A 2007, 111, 11683-11700. 
(46) Adamo, C.; Scuseria, G. E.; Barone, V. Accurate Excitation Energies from Time-Dependent Density Functional Theory: Assessing the PBE0 Model. J. Chem. Phys. 1999, 111, 28892899.

(47) TURBOMOLE V6.2 2010, a development of University of Karlsruhe and Forschungszentrum Karlsruhe GmbH, 1989-2007, TURBOMOLE GmbH, since 2007; available from http: //www.turbomole.com..

(48) Fodor, S. P. A.; Rava, R. P.; Hays, T. R.; Spiro, T. G. Ultraviolet Resonance Raman Spectroscopy of the Nucleotides With 266-, 240-, 218-, and 200-Nm Pulsed Laser Excitation. J. Am. Chem. Soc. 1985, 107, 1520-1529.

(49) Nishimura, Y.; Tsuboi, M.; Kubasek, W. L.; Bajdor, K.; Peticolas, W. L. Ultraviolet Resonance Raman Bands of Guanosine and Adenosine Residues Useful for the Determination of Nucleic Acid Conformation. J. Raman Spectrosc. 1987, 18, 221-227.

(50) Clark, L. B. Electronic Spectra of Crystalline Guanosine: Transition Moment Directions of the Guanine Chromophore. J. Am. Chem. Soc. 1994, 116, 5265-5270.

(51) Shukla, M. K.; Leszczynski, J. TDDFT Investigation on Nucleic Acid Bases: Comparison With Experiments and Standard Approach. J. Comput. Chem. 2004, 25, 768-778.

(52) Shukla, M.; Leszczynski, J. Electronic Spectra, Excited State Structures and Interactions of Nucleic Acid Bases and Base Assemblies: A Review. J. Biomol. Struct. Dyn. 2007, 25, 93118.

(53) Toyama, A.; Hanada, N.; Ono, J.; Yoshimitsu, E.; Takeuchi, H. Assignments of Guanosine UV Resonance Raman Bands on the Basis of ${ }^{13} \mathrm{C},{ }^{15} \mathrm{~N}$ and ${ }^{18} \mathrm{O}$ Substitution Effects. J. Raman Spectros. 1999, 30, 623-630.

(54) Voelter, W.; Records, R.; Bunnenberg, E.; Djerassi, C. Magnetic Circular Dichroism Studies. 
Vi. Investigation of Some Purines, Pyrimidines, and Nucleosides. J. Am. Chem. Soc. 1968, 90, 6163-6170.

(55) Mathlouthi, M.; Seuvre, A. M.; Koenig, J. L. F.T.-I.R. and Laser-Raman Spectra of Guanine and Guanosine. Carbohyd. Res. 1986, 146, 15-27.

(56) Florián, J. Scaled Quantum Mechanical Force Fields and Vibrational Spectra of Solid-State Nucleic Acid Constituents. 6. Guanine and Guanine Residue. J. Phys. Chem. 1993, 97, 10649-10658.

(57) Giese, B.; McNaughton, D. Density Functional Theoretical (DFT) and Surface-Enhanced Raman Spectroscopic Study of Guanine and Its Alkylated Derivatives. Part 1. DFT Calculations on Neutral, Protonated and Deprotonated Guanine. Phys. Chem. Chem. Phys. 2002, 4 , $5161-5170$.

(58) Tsuboi, M.; Nishimura, Y.; Hirakawa, A. Y. In Biological Applications of Raman Spectroscopy; Spiro, T. G., Ed.; Wiley: New York, 1987; Vol. 2; pp 109-179.

(59) Tannor, D. J.; Heller, E. J.; Sundberg, R. Simple Aspects of Raman Scattering. J. Phys. Chem. 1982, 86, 1822-1833. 